\title{
Fukutin Gene Mutations Cause Dilated Cardiomyopathy with Minimal Muscle Weakness
}

Terumi Murakami, MD, ${ }^{1,2}$ Yukiko K. Hayashi MD, PhD, ${ }^{1}$ Satoru Noguchi, $\mathrm{PhD}^{1,2}$ Megumu Ogawa, BS ${ }^{1}$ Ikuya Nonaka, MD, PhD, ${ }^{1}$ Yuzo Tanabe, MD, ${ }^{3}$ Mieko Ogino, MD, PhD,${ }^{4}$ Fumio Takada, MD, PhD, ${ }^{5}$ Makoto Eriguchi, $\mathrm{MD}^{6}$ Norihiko Kotooka, MD, Kevin P. Campbell, $\mathrm{PhD},{ }^{8}$ Makiko Osawa, MD, PhD, ${ }^{2}$ and Ichizo Nishino, $\mathrm{MD}, \mathrm{PhD}^{1}$

Objective: The fukutin gene (FKTN) is the causative gene for Fukuyama-type congenital muscular dystrophy, characterized by rather homogeneous clinical features of severe muscle wasting and hypotonia from early infancy with mental retardation. In contrast with the severe dystrophic involvement of skeletal muscle, cardiac insufficiency is quite rare. Fukuyama-type congenital muscular dystrophy is one of the disorders associated with glycosylation defects of $\alpha$-dystroglycan, an indispensable molecule for intra-extra cell membrane linkage.

Methods: Protein and functional analyses of $\alpha$-dystroglycan and mutation screening of FKTN and other associated genes were performed.

Results: Surprisingly, we identified six patients in four families showing dilated cardiomyopathy with no or minimal limb girdle muscle involvement and normal intelligence, associated with a compound heterozygous FKTN mutation. One patient died by rapid progressive dilated cardiomyopathy at 12 years old, and the other patient received cardiac implantation at 18 years old. Skeletal muscles from the patients showed minimal dystrophic features but have altered glycosylation of $\alpha$-dystroglycan and reduced laminin binding ability. One cardiac muscle that underwent biopsy showed altered glycosylation of $\alpha$-dystroglycan similar to that observed in a Fukuyama-type congenital muscular dystrophy patient.

Interpretation: FKTN mutations could cause much wider spectrum of clinical features than previously perceived, including familial dilated cardiomyopathy and mildest limb girdle muscular dystrophy.

Ann Neurol 2006;60:597-602

A group of disorders due to altered glycosylation of $\alpha$-dystroglycan $(\alpha-D G)$, namely, $\alpha$-dystroglycanopathy $(\alpha-D G P)$, is clinically characterized by a combination of muscular dystrophy, structural brain anomaly, and ocular involvement. ${ }^{1-7}$ Fukuyama-type congenital muscular dystrophy (FCMD) is the most common form of $\alpha$-DGP in Japan, and the patients carry the founder mutation of $3 \mathrm{~kb}$ retrotransposal insertion in the fukutin gene (FKTN), homozygously or heterozygously. ${ }^{7-9}$ Clinically, FCMD is characterized by severe congenital muscular dystrophy associated with mental retardation due to brain malformation. ${ }^{10-14}$ Most patients can speak only less than 20 meaningful words with no sentence formation. The peak motor function is seen from the age of 2 to 8 years, and their maximal motor ability is usually unassisted sitting or sliding on the buttocks. A number of the patients never acquire head control. Few patients can obtain independent ambulation, but would soon lose this ability. ${ }^{14-16}$ The prognosis is poor with their mean life span of less than 20 years. The FCMD patients with a compound heterozygous mutation of $3 \mathrm{~kb}$ insertion and missense mutation often show more severe clinical features compared with the patients with a homozygous $3 \mathrm{~kb}$ insertion mutation. ${ }^{9}$ Reportedly, there has been only two non-Japanese patients harboring null mutation in FKTN gene in both alleles. ${ }^{17,18}$ Both were Turkish boys whose clinical features were quite severe resembling Walker-Warburg syndrome, which include generalized hypotonia, hy-
From the ${ }^{1}$ Department of Neuromuscular Research, National Institute of Neuroscience, National Center of Neurology and Psychiatry; ${ }^{2}$ Department of Pediatrics, Tokyo Women's Medical University, Tokyo; ${ }^{3}$ Division of Neurology, Chiba Children's Hospital, Chiba; ${ }^{4}$ Department of Neurology, Kitasato University School of Medicine; ${ }^{5}$ Department of Medical Genetics, Kitasato University Graduate School of Medical Sciences, Kanagawa; Divisions of ${ }^{6}$ Neurology and ${ }^{7}$ Cardiovascular Medicine, Saga University Faculty of Medicine, Saga, Japan; and ${ }^{8}$ Howard Hughes Medical Institute Research Laboratories, University of Iowa College of Medicine, Iowa City, IA.
Received Aug 9, 2006, and in revised form Aug 9. Accepted for publication Aug 18, 2006.

Published online Oct 10, 2006 in Wiley InterScience (www.interscience.wiley.com). DOI: 10.1002/ana.20973

Address correspondence to Dr Hayashi, Department of Neuromuscular Research, National Institute of Neuroscience, National Center of Neurology and Psychiatry (NCNP), 4-1-1 Ogawa-Higashi, Kodaira, Tokyo 187-8502, Japan. E-mail: hayasi_y@ncnp.go.jp 


\begin{tabular}{|c|c|c|c|c|c|c|}
\hline \multirow[b]{2}{*}{ Characteristics } & \multicolumn{6}{|c|}{ Patient No. } \\
\hline & $1^{\mathrm{a}}$ & $2^{\mathrm{a}}$ & $3^{\mathrm{a}}$ & $4^{\mathrm{a}}$ & 5 & 6 \\
\hline Age (yr)/Sex & $30 / \mathrm{M}$ & $33 / \mathrm{F}$ & $22 / \mathrm{F}$ & $12 / \mathrm{M}$ & $54 / F$ & $30 / \mathrm{F}$ \\
\hline $\begin{array}{l}\text { Age at onset of } \\
\text { DCM (yr) }\end{array}$ & 17 & 20 & 11 & 12 & 46 & 30 \\
\hline $\mathrm{LVEF}^{\mathrm{b}}$ & $14 \%$ & $40 \%$ & $25 \%$ & $14 \%$ & $31 \%$ & $32 \%$ \\
\hline LVDd (mm) & 69 & 59 & 64 & 75 & 78 & 63 \\
\hline LVDs (mm) & 59 & 46 & 57 & 72 & 64 & 52 \\
\hline Age at biopsy (yr) & 18 & Not done & 8 & 12 (autopsy) & 46 & 30 \\
\hline Serum CK (IU/L) & $400-3,000$ & $1,500-1,800$ & $2,000-4,000$ & High CK & $1,200-2,000$ & 1,139 \\
\hline Motor ability & Ambulant & Ambulant & Ambulant & Ambulant & Ambulant & Ambulant \\
\hline Calf hypertrophy & Positive & Negative & Positive & Negative & Positive & Positive \\
\hline Gowers' sign & $\begin{array}{l}\text { Positive since age } \\
24 \mathrm{yr}\end{array}$ & Negative & Negative & Negative & $\begin{array}{l}\text { Positive since age } \\
41 \mathrm{yr}\end{array}$ & $\begin{array}{l}\text { Positive since } \\
26 \text { age yr }\end{array}$ \\
\hline Intelligence & Normal & Normal & Normal & Normal & Normal & Normal \\
\hline Eye abnormality & None & None & None & None & None & None \\
\hline FKTN mutation & $\begin{array}{l}\text { 3kb insertion } \\
\text { p.Q358P }\end{array}$ & $\begin{array}{l}\text { 3kb insertion } \\
\text { p.Q358P }\end{array}$ & $\begin{array}{l}3 \mathrm{~kb} \text { insertion } \\
\text { p.R179T }\end{array}$ & $\begin{array}{l}3 \mathrm{~kb} \text { insertion } \\
\text { p.R179T }\end{array}$ & $\begin{array}{l}3 \mathrm{~kb} \text { insertion } \\
\text { p.R179T }\end{array}$ & $\begin{array}{l}\text { 3kb insertion } \\
\text { p.R179T }\end{array}$ \\
\hline
\end{tabular}

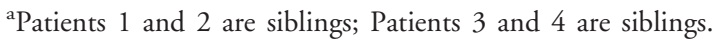

${ }^{b}$ Left ventricular ejection fraction (LVEF): normal $>60 \%$.

$\mathrm{DCM}=$ dilated cardiomyopathy; LVDd = left ventricular end-diastolic dimension; LVDs = left ventricular end-systolic dimension; CK = creatine kinase.

drocephaly, bilateral ocular abnormalities, and cataracts. They died during early infancy.

Here we report on six Japanese patients in four unrelated families with a compound heterozygous FKTN mutation presenting with dilated cardiomyopathy. All six patients show no muscle weakness until adulthood without any mental retardation. Our findings expand the phenotypic spectrum of FKTN mutations from severe congenital muscular dystrophy to dilated cardiomyopathy with mildest limb girdle muscular dystrophy.

\section{Patients and Methods}

\section{Clinical Materials}

All clinical materials used in this study were acquired with informed consent. The muscle samples were taken for diagnostic purpose and flash-frozen in isopentane chilled with liquid nitrogen.

\section{Immunohistochemistry, Immunoblotting, and Laminin Overlay Assay}

The detailed techniques of immunohistochemistry, immunoblotting, and laminin overlay assay have been described previously. ${ }^{7,19}$ The following antibodies were used for immunohistochemical and immunoblotting analyses: monoclonal anti- $\alpha$-DG (VIA4-1; Upstate Biotechnology, Lake Placid, $\mathrm{NY}$ ), polyclonal anti- $\alpha$-DG (GT20ADG), ${ }^{20}$ monoclonal anti-laminin $\alpha 2$ chain $(5 \mathrm{H} 2$; Chemicon, Temecula, CA), polyclonal anti-laminin-1 (Sigma, St. Louis, MO), and monoclonal anti- $\beta$-DG (43DAG1/8D5; Novocastra Laboratories, Newcastle upon Tyne, United Kingdom).

\section{Mutation Analyses of FKTN, FKRP, POMGnT1,} POMT1, POMT2, and LARGE

DNA was isolated from peripheral lymphocytes or muscles using a standard technique. To detect the $3 \mathrm{~kb}$ retrotransposal insertion in FKTN, we performed the genomic polymerase chain reaction using two primer sets, as described previously ${ }^{21}$; one is designed to amplify a $400 \mathrm{bp}$ product containing a part of retrotransposal insertion, and the other is designed to amplify a normal 269bp fragment. All exons and their flanking intronic regions of FKTN were sequenced directly in patients without homozygous retrotransposal insertion using an ABI PRISM 3100 automated sequencer (PE Applied Biosystems, Foster City, CA).

Mutation analysis of fukutin-related protein (FKRP), protein O-mannose $\quad \beta 1,2-N$-acetylglucosaminyltransferase 1 (POMGnT1), protein O-mannosyltransferase 1 and 2 (POMT1 and POMT2), and LARGE was also performed by directly sequencing all exons and their flanking introns. Sequences of all the primers used in this study are available on request.

\section{Results}

\section{Clinical Findings}

Detailed clinical features and mutation in FKTN of six patients from four families are described in the Table.

Patient 1 is a 30 -year-old man. He was a table tennis player with good health until 17 years of age. At this time, he started experiencing dyspnea and was diagnosed with idiopathic dilated cardiomyopathy in congestive heart failure. Echocardiography demonstrated dilated hypokinetic left ventricle with an ejection fraction of $14 \%$ (see the Table). Although he did not have 


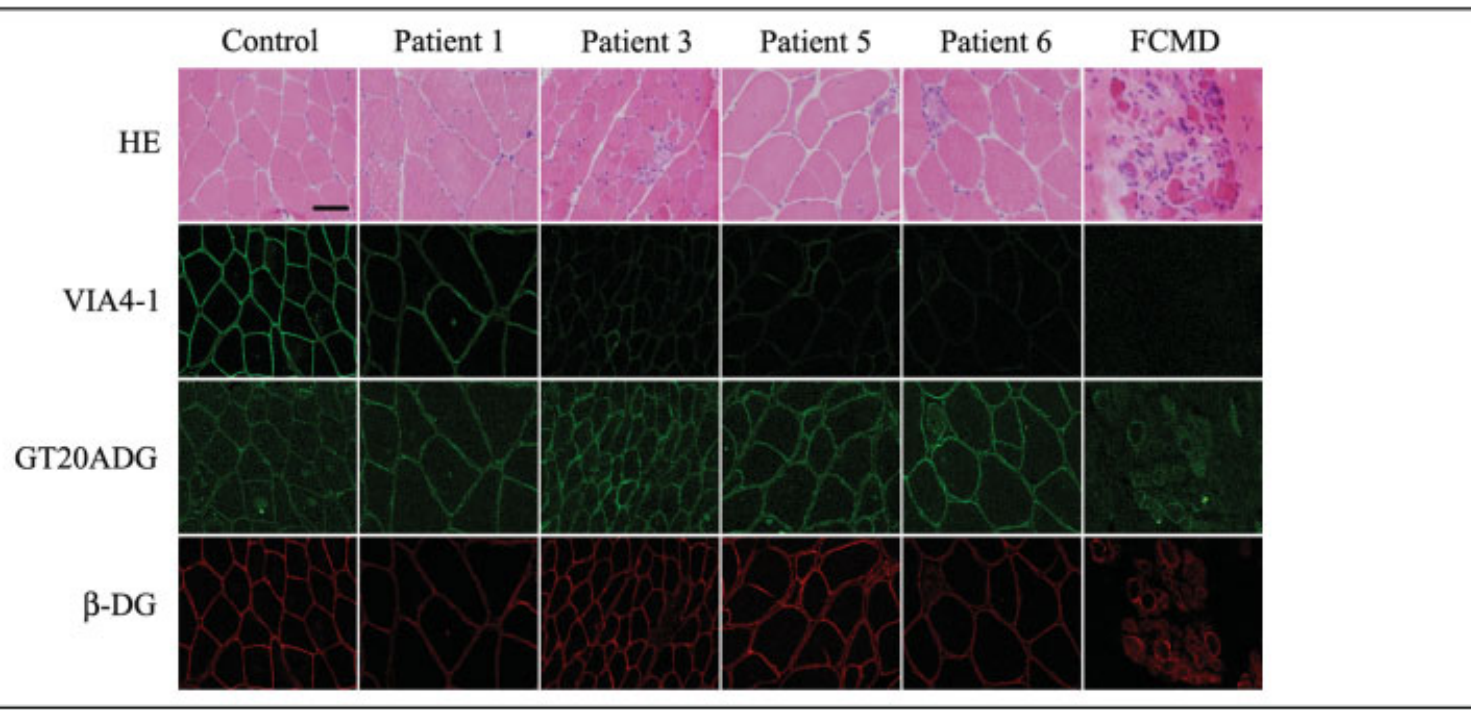

Fig 1. Hematoxylin and eosin staining (HE) and immunohistochemical analysis. On HE, mild variation in fiber size and a few regenerating fibers are seen in all four patients (Patient 1, 3, 5, and 6). Muscle from Fukuyama-type congenital muscular dystrophy (FCMD) shows severe dystrophic changes with dense deposition of fibrous and adipose tissues. Immunohistochemical analysis using antibodies VIA4-1, which recognize heavily glycosylated form of $\alpha$-dystroglycan $(\alpha-D G)$, showed reduced sarcolemmal staining in all four patients and barely detectable in FCMD compared with control muscle. Well-preserved immunoreactivities of GT20ADG for core peptide of $\alpha-D G$ and $\beta-D G$ are seen in all muscle specimens. Bar $=50 \mu \mathrm{m}$.

any muscle weakness, elevation of serum creatine kinase (CK) levels ranging from 400 to $800 \mathrm{IU} / \mathrm{L}$ (reference range, 51-197IU/L) led to muscle biopsy, showing mild variation in fiber size and internal nuclei in some fibers (Fig 1). His cardiac insufficiency progressed rapidly, necessitating heart transplantation, which he finally received at 18 years of age. After that, he had an uneventful clinical course until 24 years old when he noticed slowly progressive proximal muscle weakness of lower extremities. At 30 years of age, he shows calf hypertrophy, Gowers' sign, and mild waddling gait. Serum CK levels are elevated, ranging from 2,500 to 3,000IU/L. Muscle computed tomography shows atrophy in bilateral hamstring, gluteus, biceps brachii, and paravertebral muscles.

Patient 2 is a 33-year-old older sister of Patient 1. She enjoyed tennis during her college years. At the age of 20 , cardiomegaly was suggested by chest radiograph on health checkup. She was followed up with no medication administered. She had no clinical symptoms until 27 years of age, when she developed rapidly progressive cardiac failure during pregnancy. She underwent induced abortion. Thereafter, her cardiac functions recovered and she has remained asymptomatic even at present.

Patient 3 is a 22-year-old woman. She was born term after an uneventful pregnancy from nonconsanguineous healthy Japanese parents. Her developmental milestones were at par with age. Elevation of serum CK level was incidentally found when she had a bout of flu at 8 years old. Calf hypertrophy was likewise noticed. She subsequently underwent muscle biopsy showing a few necrotic and regenerating fibers in addition to fiber size variation (see Fig 1). At age 11, she was diagnosed to have cardiomyopathy, but she was still able to join volleyball team in her junior high school. She completed her university studies and currently is employed as an office worker. She can run, climb stairs, and does not have any limitation in her daily activities. She has no muscle weakness including facial muscles, but has calf hypertrophy. She does not have scoliosis and joint contractures. Serum CK levels are elevated from 2,000 to 4,000IU/L. Electrocardiogram shows right bundle branch block. Echocardiography shows left ventricular enlargement with decreased ejection fraction, suggestive of dilated cardiomyopathy. Despite detailed ophthalmological examinations, no abnormalities were found. Muscle computed tomography suggests mild fatty infiltration in hamstrings, gastrocnemius, and gluteus maximum muscles. Brain magnetic resonance imaging is normal except for one cerebellar cyst and probable focal pachygyria in the left occipital lobe.

Patient 4, older brother of Patient 3, was healthy and did not have either muscle weakness or calf hypertrophy. He was a swimmer during his early childhood. At 12 years old, he developed progressive dyspnea and was subsequently diagnosed to have cardiomyopathy. Despite intensive treatments, he died of cardiac failure after 1 month. Autopsy showed severe dilated cardiomyopathy with lymphocytic infiltration and fibrosis.

Patient 5 is a 54-year-old housewife. She was born 
after an uneventful pregnancy from nonconsanguineous healthy Japanese parents. She had normal developmental milestones. At 40 years old, premature beats were observed on electrocardiogram. She also noticed difficulty in squatting. When she was 46 years old, she was admitted to a hospital because of dyspnea and was diagnosed to have dilated cardiomyopathy by echocardiography and endomyocardial biopsy. Mild proximal dominant muscle weakness without facial muscle involvement was also observed. Deep-tendon reflexes were normal. Serum CK level was elevated to 825IU/L. Muscle biopsy showed mild dystrophic changes with variation in fiber size, fibers with internal nuclei, and a few regenerating fibers (see Fig 1).

Patient 6 is a 30 -year-old woman. She was a slow runner with calf hypertrophy since childhood, but no apparent muscle weakness was experienced. At 26 years old, she had an uneventful pregnancy. Postpartum, she noticed difficulties in walking while holding her baby. At 29 years old, she became unable to walk for long distances. At 30 years old, she was diagnosed with dilated cardiomyopathy from the results of echocardiography and an endomyocardial biopsy, which showed mild fibrosis with lymphocytic infiltration. She also developed proximal dominant muscle weakness and waddling gait. Serum CK level was increased to 1,139IU/L. Muscle biopsy showed mild dystrophic changes with a few necrotic fibers and mild fiber size variation (see Fig 1).

\section{Immunohistochemical, Immunoblotting Analyses, and Laminin Overlay Assay}

Immunohistochemical analysis was performed using biopsied skeletal muscles. Immunoreaction of $\alpha-D G$ (VIA4-1), which recognizes heavily glycosylated form of $\alpha$-DG, demonstrated reduction of sarcolemmal staining from all four patients, whereas the antibody for the core peptide of $\alpha$-DG (GT20ADG) and $\beta$-DG showed well-preserved membrane staining (see Fig 1). Muscle specimens from FCMD patients showed barely detectable immunoreaction for $\alpha-D G$ (VIA4-1), as described previously (see Fig 1). ${ }^{1}$ No difference was seen between muscles from FCMD patients with a homozygous $3 \mathrm{~kb}$ insertion and more severe patients with a compound heterozygous mutation of missense and $3 \mathrm{~kb}$ insertion in FKTN. On immunoblotting analysis using VIA4-1, skeletal muscles from the four patients showed a fainter and smaller sized band than control muscle, whereas a muscle sample from FCMD showed no detectable band, as reported previously (Fig 2A). ${ }^{1}$ By using GT20ADG, muscles from patients showed fainter broad bands, whose molecular masses were bigger than the approximately $90 \mathrm{kDa}$ band observed in FCMD muscles having either homozygous or compound heterozygous mutation. A $43 \mathrm{kDa}$ immunore-

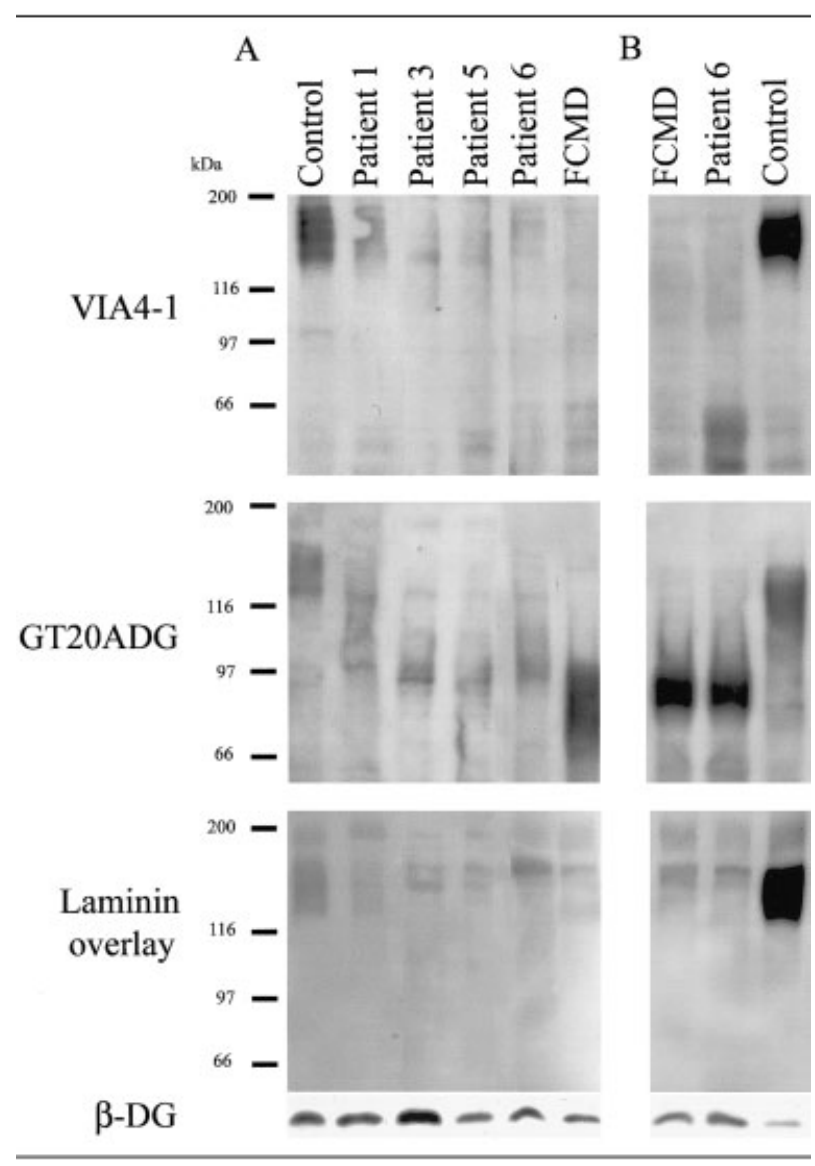

Fig 2. Immunoblotting analysis. (A) Immunoblotting analysis using antibodies of VIA4-1, GT20ADG, and $\beta$-dystroglycan $(\beta-D G)$ and laminin overlay assay are performed using skeletal muscle from control, four patients (Patients 1, 3, 5, and 6), and Fukuyama-type congenital muscular dystrophy (FCMD). Both antibodies for $\alpha-D G$ (VIA4-1 and

GT20ADG) recognize a broad band about $156 \mathrm{kDa}$ in size in control muscle. In the four patients' muscles, $\alpha-D G$ bands are reduced in size and amount compared with control. No detectable band is seen in FCMD muscle by VIA4-1; a band at approximately 90kDa was detected by GT20ADG. Laminin overlay assay shows greatly reduced but positive binding ability in the four patients, whereas it is not detectable in FCMD muscle. (B) Immunoblotting analysis of the biopsied cardiac muscle from Patient 6, autopsied control, and FCMD. Both Patient 6 and FCMD show similar reduced sized (approximately $90 \mathrm{kDa}$ ) band when compared with control.

active band for $\beta$-DG showed no difference between samples. Laminin overlay assay showed reduced but positive binding ability of $\alpha-D G$ to laminin in all four patients.

Immunoblotting analysis of biopsied cardiac muscle from Patient 6 showed no detectable band for $\alpha$-DG by VIA4-1, whereas an approximately $90 \mathrm{kDa}$ band was observed by GT20ADG, which is similar to that observed in an autopsied FCMD heart carrying a homozygous $3 \mathrm{~kb}$ insertion in FKTN. Laminin overlay as- 
say showed no detectable band in both Patient 6 and FCMD (see Fig 2B).

\section{Mutation Analysis}

All patients had a compound heterozygous mutation of $3 \mathrm{~kb}$ retrotransposal insertion and missense mutation in FKTN (see the Table). Patients 1 and 2 had the same compound heterozygous mutation of $3 \mathrm{~kb}$ insertion and c.1073A $>$ C (p.Q358P). Their healthy mother had a $3 \mathrm{~kb}$ insertion, and the healthy father carries p.Q358P mutation, in heterozygous mode. Patients 3, 4, 5, and 6 had the same compound heterozygous mutation of $3 \mathrm{~kb}$ insertion and c.536G $>\mathrm{C}$ (p.R179T). The father of Patients 3 and 4 had a $3 \mathrm{~kb}$ insertion, and the mother had a p.R179T mutation, both in heterozygous mode. These two missense mutations were not detected in 100 chromosomes from Japanese healthy control individuals. The amino acids of both mutated positions are highly conserved among various species including chimpanzee, cow, mouse, rat, chicken, and fish (data not shown).

No mutation was found in the other responsible genes for $\alpha$-DGP including FKRP, POMGnT1, POMT1, POMT2, and LARGE in these patients.

\section{Discussion}

FCMD is one of the most severe congenital muscular dystrophies with central nervous system involvement. Severe mental retardation and epilepsy are characteristic clinical features of FCMD, with brain showing polymicrogyria/pachygyria caused by altered neuronal migration. Muscle weakness is severe, and the patients show floppiness from infancy. Most patients cannot achieve independent ambulation and became bedridden by the second decade of life. Marked dystrophic changes of skeletal muscle with dense deposition of fibrous and adipose tissues are characteristic since early infancy. In contrast with the severely affected skeletal muscle, clinical manifestations of cardiac impairment are quite rare. Only a few patients were reported to have cardiac insufficiency, although fibrosis of myocardium is frequently seen in the autopsy studies. ${ }^{22}$

The clinical features of the patients reported here are completely different from those seen in FCMD. All six patients show quite normal intelligence with no epileptic episodes. Muscle weakness is extremely mild, if any, and appears only in adulthood. Muscle computed tomography/magnetic resonance imaging showed mild signal changes in posterior thigh and hip muscles. These findings are also dissimilar from FCMD patients, because calf muscles are more severely affected than thigh muscles in FCMD patients. ${ }^{16}$ Pathological findings in the biopsied skeletal muscles showed only minimal dystrophic changes, which, again, is quite different from FCMD.

$\alpha-D G$ is a highly glycosylated surface membrane protein, and its molecular mass in normal skeletal muscle is about $156 \mathrm{kDa}$. In FCMD muscles, regardless the type of mutation or clinical severity, altered glycosylation of $\alpha$-DG reduces its molecular mass to approximately $90 \mathrm{kDa}$, resulting in a lack of laminin binding ability. ${ }^{1,20}$ Biopsied muscles from the patients described here, however, showed larger sized $\alpha$-DG than FCMD muscles with reduction of laminin binding ability. These findings are similar to those observed in muscles from mild limb girdle muscular dystrophy type 2I (LGMD2I) patients caused by mutations in FKRP. ${ }^{2}$ Minimal muscle involvement, if any, with partly glycosylated $\alpha-\mathrm{DG}$ suggests that the mutated fukutin may preserve part of its function in skeletal muscle.

Cardiac involvement is the most remarkable finding in our patients. All the patients showed dilated cardiomyopathy, and two of them had life-threatening, rapidly progressive cardiac insufficiency. Cardiac involvement is rarely described in patients with $\alpha$-DGP except for some patients with LGMD2I. ${ }^{23-25}$ In these patients, cardiac symptoms are not always correlated with severity of skeletal muscle weakness, and one family showed dilated cardiomyopathy without any muscular dystrophy. ${ }^{26}$ It is unclear why the severity of affected organs is different, but one possible explanation is that the patients with no or minimal muscle weakness can run and perform exercise as normal individuals. Vigorous physical activities could induce stress and accelerate degeneration of cardiac muscles, resulting in progressive cardiomyopathy. The difference of glycosylation of $\alpha$-DG between cardiac and skeletal muscles is also notable, although detailed composition of the sugar moiety in each tissue is still unknown. In contrast with the skeletal muscle, the molecular mass of $\alpha$-DG in the biopsied cardiac muscle from Patient 6 was similar to that from FCMD patient. The mutant fukutin caused by missense mutations in FKTN identified in this series may have a different effect on glycosylation of $\alpha-D G$ between skeletal and cardiac muscles. To date, the molecular mechanism of cardiomyopathy associated with FKTN mutation is still unclear. Further extensive analyses are needed to clarify the roles of fukutin in the glycosylation process of $\alpha$-DG in the different organs.

Our data indicate that the clinical spectrum caused by FKTN mutations is much wider than previously perceived, from the most severe form of congenital muscular dystrophy with central nervous system involvement to dilated cardiomyopathy with mildest limb girdle muscular dystrophy. Careful follow-up of cardiac function is necessary for the patients with $\alpha$-DGP. More importantly, patients with cardiomyopathy should also be examined for skeletal muscle involvement, including determination of serum CK levels. Several responsible genes for dilated cardiomyopathy have been identified; however, mutations are successfully identified in only a fraction of the patients. 
It is important to consider the mutation in FKTN for diagnosis of familial dilated cardiomyopathy, although it is rarely found outside of Japan.

This study was supported by the Research on Health Sciences focusing on Drug Innovation from the Japanese Health Sciences Foundation (SH4409, I.N.), the Research on Psychiatric and Neurological Diseases and Mental Health of Health and Labor Sciences Research Grants (H16-020, I.N.), the Research Grant (17A-10, I.N.) for Nervous and Mental Disorders from the Ministry of Health, Labor and Welfare, the Grant-in-Aid for Scientific Research from the Japan Society for the Promotion of Science (16209029, I.N.), and the Program for Promotion of Fundamental Studies in Health Sciences of the National Institute of Biomedical Innovation (NIBIO, 05-32, I.N.).

We are grateful to Dr T. Inomata and E. Watanabe for the detailed clinical information. We also thank M. Ogawa for technical assistance and Dr M. Astejada for reviewing the manuscript.

\section{References}

1. Hayashi YK, Ogawa M, Tagawa K, et al. Selective deficiency of alpha-dystroglycan in Fukuyama-type congenital muscular dystrophy. Neurology 2001;57:115-121.

2. Brockington $M$, Blake DJ, Prandini $P$, et al. Mutations in the fukutin-related protein gene (FKRP) cause a form of congenital muscular dystrophy with secondary laminin alpha2 deficiency and abnormal glycosylation of alpha-dystroglycan. Am J Hum Genet 2001;69:1198-1209.

3. Beltran-Valero de Bernabe D, Currier S, Steinbrecher A, et al. Mutations in the $\mathrm{O}$-mannosyltransferase gene POMT1 give rise to the severe neuronal migration disorder Walker-Warburg syndrome. Am J Hum Genet 2002;71:1033-1043.

4. Kano H, Kobayashi K, Herrmann R, et al. Deficiency of alphadystroglycan in muscle-eye-brain disease. Biochem Biophys Res Commun 2002;291:1283-1286.

5. Muntoni F, Brockington M, Blake DJ, et al. Defective glycosylation in muscular dystrophy. Lancet 2002;360:1419-1421.

6. Kim DS, Hayashi YK, Matsumoto H, et al. POMT1 mutation results in defective glycosylation and loss of laminin-binding activity in alpha-DG. Neurology 2004;62:1009-1011.

7. Matsumoto H, Hayashi YK, Kim DS, et al. Congenital muscular dystrophy with glycosylation defects of alpha-dystroglycan in Japan. Neuromuscul Disord 2005;15:342-348.

8. Kobayashi K, Nakahori Y, Miyake M, et al. An ancient retrotransposal insertion causes Fukuyama-type congenital muscular dystrophy. Nature 1998;394:388-392.

9. Kondo-Iida E, Kobayashi K, Watanabe M, et al. Novel mutations and genotype-phenotype relationships in 107 families with Fukuyama-type congenital muscular dystrophy (FCMD). Hum Mol Genet 1999;8:2303-2309.
10. Fukuyama Y, Kawazura M, Haruna H. A peculier form of congenital progressive muscular dystrophy: report of fifteen cases. Paediatr Univ Tokyo 1960;4:5-8.

11. Fukuyama Y, Osawa M, Suzuki H. Congenital progressive muscular dystrophy of the Fukuyama type: clinical, genetic and pathological considerations. Brain Dev 1981;3:1-29.

12. Fukuyama Y, Ohsawa M. A genetic study of the Fukuyama type congenital muscular dystrophy. Brain Dev 1984;6: 373-390.

13. Osawa M, Arai Y, Ikenaka H, et al. Fukuyama type congenital progressive muscular dystrophy. Acta Paediatr Jpn 1991;33: 261-269.

14. Kondo-Iida E, Saito K, Tanaka H, et al. Molecular genetic evidence of clinical heterogeneity in Fukuyama-type congenital muscular dystrophy. Hum Genet 1997;99:427-432.

15. Saito K, Osawa M, Wang ZP, et al. Haplotype-phenotype correlation in Fukuyama congenital muscular dystrophy. Am J Med Genet 2000;92:184-190.

16. Osawa M, Wang ZP, Saito K, et al. Clinicopathological study of transiently ambulant Fukuyama-type congenital muscular dystrophy cases. Acta Myol 2001;20:96-103.

17. Silan F, Yoshioka M, Kobayashi K, et al. A new mutation of the fukutin gene in a non-Japanese patient. Ann Neurol 2003; 53:392-396.

18. de Bernabe DB, van Bokhoven $\mathrm{H}$, van Beusekom E, et al. A homozygous nonsense mutation in the fukutin gene causes a Walker-Warburg syndrome phenotype. J Med Genet 2003;40: $845-848$

19. Hayashi YK, Engvall E, Arikawa-Hirasawa E, et al. Abnormal localization of laminin subunits in muscular dystrophies. J Neurol Sci 1993;119:53-64.

20. Michele DE, Barresi R, Kanagawa M, et al. Post-translational disruption of dystroglycan-ligand interactions in congenital muscular dystrophies. Nature 2002;418:417-422.

21. Kato R, Kawamura J, Sugawara H, et al. A rapid diagnostic method for a retrotransposal insertional mutation into the FCMD gene in Japanese patients with Fukuyama congenital muscular dystrophy. Am J Med Genet A 2004;127:54-57.

22. Osawa M, Sumida S, Suzuki Y, et al. Fukuyama type congenital progressive muscular dystrophy. In: Fukuyama Y, Osawa M, Saito K, eds. Congenital muscular dystrophies. Amsterdam: Elsevier Science BV, 1997:31-68.

23. Poppe M, Cree L, Bourke J, et al. The phenotype of limb-girdle muscular dystrophy type 2I. Neurology 2003;60:1246-1251.

24. Mercuri E, Brockington M, Straub V, et al. Phenotypic spectrum associated with mutations in the fukutin-related protein gene. Ann Neurol 2003;53:537-542.

25. Walter MC, Petersen JA, Stucka R, et al. FKRP (826C>A) frequently causes limb-girdle muscular dystrophy in German patients. J Med Genet 2004;41:e50.

26. Muller T, Krasnianski M, Witthaut R, et al. Dilated cardiomyopathy may be an early sign of the C826A Fukutin-related protein mutation. Neuromuscul Disord 2005;15:372-376. 